EPJ manuscript No.

(will be inserted by the editor)

\title{
Renormalized Parameters for Impurity Models
}

\author{
A.C.Hewson ${ }^{1}$, A. Oguri ${ }^{2}$, and D. Meyer ${ }^{1}$ \\ 1 Department of Mathematics, Imperial College, London SW7 2BZ, UK \\ 2 Department of Material Science, Osaka City University, Sumiyoshi-ku, Osaka 558-8585 Japan
}

Received: November 6, 2018

\begin{abstract}
We show that the low energy behaviour of quite diverse impurity systems can be described by a single renormalized Anderson model, with three parameters, an effective level $\tilde{\epsilon}_{d}$, an effective hybridization $\tilde{V}$, and a quasiparticle interaction $\tilde{U}$. The renormalized parameters are calculated as a function of the bare parameters for a number of impurity models, including those with coupling to phonons and a FalikovKimball interaction term. In the model with a coupling to phonons we determine where the interaction of the quasiparticles changes sign as a function of the electron-phonon coupling. In the model with a FalikovKimball interaction we show that to a good approximation the low energy behaviour corresponds to that of a bare Anderson model with a shifted impurity level.
\end{abstract}

PACS. 71.10.-w Theories and models of many-electron systems - 71.27.+a Strongly correlated electron systems; heavy fermions - 75.20.Hr Local moment in compounds and alloys; Kondo effect, valence fluctuations, heavy fermions

\section{Introduction}

The low energy behaviour of impurities in a metallic host can be calculated using a numerical renormalization group (NRG) approach in which the higher energy states are progressively eliminated 12 . The low temperature ther' modynamics of the impurity are deduced from the leading corrections to the low energy fixed point of the renormalization group transformation. An alternative approach to the calculation of the low energy behaviour is the renormalized perturbation theory (RPT) 3 . When this ap' proach is applied to the Anderson impurity model, for instance, the effective low energy model generated is just a renormalized version of the Anderson model; expressions for the renormalized parameters can be derived in terms of the self-energy, its derivative and the irreducible four-vertex, all evaluated at zero frequency 34. In this paper we will clarify the relation between these two approaches, and consider the various possible ways of determining the renormalized parameters. We will also generalize the approach to include models which have a coupling to phonons and a Falikov-Kimball screening interaction between the impurity and conduction electrons.

The Anderson model [5] has the form,

$$
\begin{aligned}
H_{\mathrm{AM}}= & \sum_{\sigma} \epsilon_{d} d_{\sigma}^{\dagger} d_{\sigma}+U n_{d, \uparrow} n_{d, \downarrow}+\sum_{k, \sigma} \epsilon_{k, \sigma} c_{k, \sigma}^{\dagger} c_{k, \sigma}+ \\
& +\sum_{k, \sigma}\left(V_{k} d_{\sigma}^{\dagger} c_{k, \sigma}+V_{k}^{*} c_{k, \sigma}^{\dagger} d_{\sigma}\right) .
\end{aligned}
$$

It describes a localized level $\epsilon_{d}$ of an impurity, hybridized with the conduction electrons of the host metal via the matrix element $V_{k}$. There is in addition a local interaction $U$ between the electrons on the impurity site. When $U=0$ the local level broadens into a resonance, corresponding to a localized quasi-bound state, whose width depends on the quantity $\Delta(\omega)=\pi \sum_{k}\left|V_{k}\right|^{2} \delta\left(\omega-\epsilon_{k}\right)$. It is usual to consider the case of a wide conduction band with a flat density of states where $\Delta(\omega)$ becomes independent of $\omega$ and can be taken as a constant $\Delta$.

In the renormalized model used in the RPT approach there is an effective energy level at $\tilde{\epsilon}_{d}$, and an effective resonance width $\tilde{\Delta}$. Expressions for these renormalized parameters can be derived in terms of the 'bare' parameters, $\epsilon_{d}, \Delta$, the local self-energy $\Sigma_{\sigma}(\omega, h)$ and its frequency derivative $\Sigma_{\sigma}^{\prime}(\omega, h)$ evaluated at zero frequency $\omega=0$, zero magnetic field $h=0$, and $T=0$. They are given by

$$
\tilde{\epsilon}_{\mathrm{d}}=z\left(\epsilon_{\mathrm{d}}+\Sigma_{\sigma}(0,0)\right), \quad \tilde{\Delta}=z \Delta,
$$

where $z$, the wavefunction renormalization factor, is given by $z=1 /\left(1-\Sigma_{\sigma}^{\prime}(0,0)\right)$. The effective local interaction $\tilde{U}$ is expressed in terms of the local four-vertex $\Gamma_{\uparrow, \downarrow}\left(\omega_{1}, \omega_{2}, \omega_{3}, \omega_{4}\right)$ evaluated at zero frequency $\left(\omega_{1}=\omega_{2}=\omega_{3}=\omega_{4}=0\right)$,

$$
\tilde{U}=z^{2} \Gamma_{\uparrow, \downarrow}(0,0,0,0) .
$$

The renormalized effective model has the same form as (1), but in terms of renormalized parameters, and the interaction term is normal-ordered, as it only comes into play when two or more excitations are created relative to 
the ground state of the interacting system,

$$
\begin{aligned}
\tilde{H}_{\mathrm{AM}}= & \sum_{\sigma} \tilde{\epsilon}_{d} d_{\sigma}^{\dagger} d_{\sigma}+\tilde{U}: n_{d, \uparrow} n_{d, \downarrow}:+\sum_{k, \sigma} \epsilon_{k, \sigma} c_{k, \sigma}^{\dagger} c_{k, \sigma}+ \\
& +\sum_{k, \sigma}\left(\tilde{V}_{k} d_{\sigma}^{\dagger} c_{k, \sigma}+\tilde{V}_{k}^{*} c_{k, \sigma}^{\dagger} d_{\sigma}\right)
\end{aligned}
$$

where the colon brackets indicate that the expression within them must be normal-ordered. This renormalized model is similar to that used in earlier phenomenological local Fermi-liquid theories [6], but here it also includes a quasiparticle interaction term.

In the renormalized perturbation theory the Hamiltonians of the bare and renormalized Anderson models are related via $H_{\mathrm{AM}}=\tilde{H}_{\mathrm{AM}}+\tilde{H}_{c}$, where $\tilde{H}_{c}$ is the counterterm Hamiltonian given by

$$
\tilde{H}_{c}=\sum_{\sigma} \lambda_{1} d_{\sigma}^{\dagger} d_{\sigma}+\lambda_{2} n_{d, \uparrow} n_{d, \downarrow} .
$$

The renormalized perturbation expansion is in powers of the renormalized interaction $\tilde{U}$, but all the terms in (4) and (5) are taken into account; the parameters $\lambda_{1}$ and $\lambda_{2}$, and a rescaling factor $\lambda_{3}$, are determined by the condition that there is no further renormalization of the already fully renormalized parameters, $\tilde{\epsilon}_{d}, \tilde{\Delta}$ and $\tilde{U}$ arising from the expansion (the procedure is clearer in the Lagrangian formulation, for details see [4]). It has been shown [3 that the normal-ordered renormalized Anderson model (44) is sufficient for the calculation of thermodynamic properties in the low energy, low field, and low temperature regime. The counter-term part of the Hamiltonian need only be taken into account explicitly for the calculation of corrections for higher temperatures and magnetic fields 4]. The first order expressions for the impurity spin and charge susceptibilities, $\chi_{s, \text { imp }}$ and $\chi_{c, \text { imp }}$ at $T=0$ derived from the renormalized model are exact [7] and given by

$$
\begin{aligned}
& \chi_{s, \mathrm{imp}}=\frac{1}{2} \tilde{\rho}_{\mathrm{imp}}(0)\left(1+\tilde{U} \tilde{\rho}_{\mathrm{imp}}(0)\right), \\
& \chi_{c, \mathrm{imp}}=\frac{1}{2} \tilde{\rho}_{\mathrm{imp}}(0)\left(1-\tilde{U} \tilde{\rho}_{\mathrm{imp}}(0)\right),
\end{aligned}
$$

where the spin susceptibility is given in units of $\left(g \mu_{\mathrm{B}}\right)^{2}$ and the charge susceptibility differs by a factor of $\frac{1}{4}$ from the usual definition, so it is the isospin equivalent of $\chi_{s, \mathrm{imp}}$. The quasiparticle density of states at the Fermi level $\tilde{\rho}_{\text {imp }}(0)$ is given by

$$
\tilde{\rho}_{\mathrm{imp}}(0)=\frac{\tilde{\Delta} / \pi}{\tilde{\epsilon}_{d}^{2}+\tilde{\Delta}^{2}}
$$

Exact results for specific heat coefficient $\gamma_{\mathrm{imp}}$ and the occupation of the impurity level $n_{\mathrm{imp}, \sigma}$ are

$$
\gamma_{\mathrm{imp}}=\frac{2 \pi^{2}}{3} \tilde{\rho}_{\mathrm{imp}}(0)
$$

and

$$
n_{\mathrm{imp}, \sigma}=\frac{1}{2}-\frac{1}{\pi} \tan ^{-1}\left(\frac{\tilde{\epsilon}_{\mathrm{d}}}{\tilde{\Delta}}\right)
$$

which corresponds to the Friedel sum rule 8 . These two results correspond to a local Fermi-liquid theory with noninteracting quasiparticles and can be deduced from the zero order $(U=0)$ renormalized model. The temperature dependence of the impurity contribution to the conductivity $\sigma(T)$ has also been calculated for the symmetric model to order $T^{2}$ from the renormalized self-energy calculated to second order in $\tilde{U}$. The result is

$$
\sigma(T)=\sigma_{0}\left\{1+\frac{\pi^{2}}{3}\left(\frac{T}{\tilde{\Delta}}\right)^{2}\left[1+2\left(\frac{\tilde{U}}{\pi \tilde{\Delta}}\right)^{2}\right]+\mathrm{O}\left(T^{4}\right)\right\} .
$$

When the renormalized parameters are expressed in terms of the self-energy and the vertex function these results coincide with the exact expressions derived by Yamada 9 from an analysis of perturbation theory to all orders $U$, and the Fermi-liquid results of Nozières [10] in the Kondo limit. This result has also been generalized to include the leading non-linear correction to the differential conductance through a quantum dot in the Fermi liquid regime [11, which is of order $V_{e}^{2}$, where voltage $V_{e}$ is the voltage difference across the dot. However, the values of the renormalized parameters $\tilde{\epsilon}_{d}, \tilde{\Delta}$ and $\tilde{U}$, have to be determined. In the localized or Kondo limit they can be reduced to one single parameter, the Kondo temperature $T_{\mathrm{K}}$. For $U \gg\left|\epsilon_{d}\right|$, and $\epsilon_{d} \ll 0$, the electron in the dstate at the impurity site will be localized and as a consequence the impurity charge susceptibility must vanish in this limit. From equations, (6) and (9), this implies $\tilde{U}=\pi \tilde{\Delta}$ and $\tilde{\epsilon}_{d}=0$. If the impurity susceptibility at $T=0$ is expressed in terms of a Kondo temperature $T_{\mathrm{K}}$ defined by $\chi_{s, \mathrm{imp}}=1 / 4 T_{\mathrm{K}}$, then the renormalized parameters can be expressed in terms of a single energy scale $T_{\mathrm{K}}$, so $\tilde{U}=\pi \tilde{\Delta}=4 T_{\mathrm{K}}$, and the Wilson or $\chi / \gamma$ ratio, $R=1+\tilde{U} \tilde{\rho}_{d}(0)=2$ [10].

It is clear that the renormalized Anderson model derived in the RPT must be directly related to the low energy effective model obtained in the numerical renormalization group (NRG) calculations by Wilson [1 for the s-d (Kondo) model, and by Krishnamurthy, Wilkins and Wilson (KWW) 2] for the Anderson model. The RPT and NRG approaches are, however, rather different. In the Wilson approach the conduction band of the Anderson model is replaced by a discrete spectrum of states, which is then expressed in the form of a tight-binding chain with the impurity at one end. The Hamiltonian for the discrete model for a finite chain with $N+2$ sites, including the impurity site, is

$$
\begin{aligned}
H_{\mathrm{AM}}^{N} & =\sum_{\sigma} \epsilon_{d} d_{\sigma}^{\dagger} d_{\sigma}+U n_{d, \uparrow} n_{d, \downarrow}+V \sum_{\sigma}\left(d_{\sigma}^{\dagger} c_{0, \sigma}+c_{0, \sigma}^{\dagger} d_{\sigma}\right) \\
& +\sum_{n=0, \sigma}^{n=N} \Lambda^{-n / 2} \xi_{n}\left(c_{n, \sigma}^{\dagger} c_{n+1, \sigma}+c_{n+1, \sigma}^{\dagger} c_{n, \sigma}\right),
\end{aligned}
$$

where $\Lambda>1$ is the discretization parameter, and $\xi_{n}$ is given by

$$
\xi_{n}=\frac{D}{2} \frac{\left(1+\Lambda^{-1}\right)\left(1-\Lambda^{-n-1}\right)}{\left(1-\Lambda^{-2 n-1}\right)^{1 / 2}\left(1-\Lambda^{-2 n-3}\right)^{1 / 2}},
$$


and $2 D$ is the width of the conduction band [1. The discretization of the conduction band is logarithmic and such that the density of levels increases as the Fermi-level is approached, and $\epsilon=0$ is the limit point of the sequence. With the model in this form an iterative diagonalization scheme is then set up, starting with the impurity site, and an extra site along the chain is added at each iteration step. After five or six steps the matrices become too large to handle, and after this point the Hibert space is truncated, with the higher energy states being neglected. A fixed number, of the order 500-1500 of the lowest lying energy states, is retained at each subsequent step. The couplings along the chain fall off as $\Lambda^{-n / 2}$, where $n$ is the nth site as $\Lambda>1$. Lower and lower energy scales are reached in the process but the Hamiltonian is rescaled after each step by a factor $\Lambda^{1 / 2}$, so that the lowest energy scale is formally the same after each iteration.

A renormalization group transformation can be set up in which the states and couplings can be compared after each step. This renormalization group transformation for the s-d (Kondo) and Anderson model has a low energy fixed point corresponding to states of a free chain uncoupled from the impurity. In the s-d case this was interpreted as a $J \rightarrow \infty$ fixed point, so that the impurity and first site become essentially uncoupled from the chain. As this fixed point Hamiltonian corresponds to a free chain it gives no finite contribution to the impurity susceptibility and specific heat. The finite impurity susceptibility and specific heat arise from the leading irrelevant terms of the renormalization group transformation, which correspond to a residual interaction and a one-body coupling term at the end of this decoupled chain.

Similar results hold for the Anderson model [2] which for the symmetric model can be interpreted as a $V \rightarrow \infty$ fixed point. However, from the Bethe ansatz exact solution of the Anderson model 1213 we know that the Anderson model behaves as a Fermi-liquid, whatever the value of the interaction term, so the nature of the fixed point is independent of $U$. It seems appropriate from a Fermiliquid point of view, therefore, to base the interpretation of the low energy fixed point on the original chain including the impurity, but with renormalized parameters. In this approach the fixed point and leading irrelevant terms are those of the renormalized Anderson model; this then makes a direct connection with the effective model used in the renormalized perturbation theory. It also has the advantage that it can be interpreted in terms of a quasiparticle picture in 1-1 comparison with the original model. We can use the NRG technique to calculate the renormalized Fermi-liquid parameters. A knowledge of how these renormalized parameters, such as the quasiparticle interaction $\tilde{U}$, depend on the bare parameters of the model can give us considerable insight into the low energy behaviour of impurity systems. The numerical results for the physical behaviour of the model at low temperatures, the susceptibilities, specific heat coefficient etc, are essentially the same as those obtained in the earlier NRG calculations of KWW [2]; the difference here is that they are interpreted in terms of a single renormalized model, with parameters in 1-1 correspondence with those of the original Anderson model, and the calculational procedure has also been considerably simplified. The approach can be extended to more general impurity models including orbital degeneracy [14.

In the next section of this paper we give a new way of analysing the fixed point to determine the renormalized parameters, which is straight forwardly applicable to systems with a non-symmetric and non-constant density of states. The approach is then applied to generalizations of the model to include an interaction with phonons, and Falikov-Kimball terms, for which there are in general no exact results. We examine how the quasiparticle interaction and renormalized parameters vary according to the parameter regimes of these more general impurity models. In the conclusion we discuss the question of the extent to which these additional interactions, such as those due to phonons and the Falikov-Kimball terms, may be absorbed by modifying the parameters of the bare model, or whether to describe fully the behaviour in higher energy regimes, these terms have to be included explicitly.

\section{Calculation of Renormalized Parameters}

In this section we will examine the low energy NRG fixed point of the Anderson model as a renormalized version of the same model and deduce the renormalized parameters $\tilde{\epsilon}_{d}, \tilde{\Delta}$ and $\tilde{U}$. The starting point of the NRG calculation is the discretized form of the model given in equation (III). The many-body states are calculated using the iteration procedure as outlined in the previous section. For a given $N$, we denote the minimum energy required to add a single electron to the ground state by $E_{p}(N)$, and and the minimum energy to create a single hole by $E_{h}(N)$. If these single particle and hole excitations correspond to a renormalized Anderson model, then asymptotically they should coincide with those of the free model with an appropriate choice of $\tilde{\epsilon}_{d}$ and $\tilde{\Delta}$ (the term in $\tilde{U}$ plays a role only when more than one single particle excitation is created from the ground state). This problem is considered in the appendix where we define $N$-dependent quantities, $\tilde{\epsilon}_{d}(N)$, and $\tilde{\Delta}(N)=\pi \tilde{V}(N)^{2} / 2$, via the two equations,

$$
\begin{aligned}
& \frac{\pi E_{p}(N) \Lambda^{-(N-1) / 2}}{2 \tilde{\Delta}(N)}-\frac{\pi \tilde{\epsilon}_{d}(N)}{2 \tilde{\Delta}(N)}=\Lambda^{(N-1) / 2} g_{00}\left(E_{p}(N)\right) \\
& \frac{-\pi E_{h}(N) \Lambda^{-(N-1) / 2}}{2 \tilde{\Delta}(N)}-\frac{\pi \tilde{\epsilon}_{d}(N)}{2 \tilde{\Delta}(N)}=\Lambda^{(N-1) / 2} g_{00}\left(-E_{h}(N)\right)
\end{aligned}
$$

The renormalized parameters $\tilde{\epsilon}_{d}$ and $\tilde{\Delta}$ corresponding to the low energy fixed point are given by $\tilde{\epsilon}_{d}=\lim _{N \rightarrow \infty} \tilde{\epsilon}_{d}(N)$ and $\tilde{\Delta}=\lim _{N \rightarrow \infty} \tilde{\Delta}(N)$ and are given by equations (41) and (42) in the appendix.

In figure 1 we plot the quantities $\tilde{\Delta}(N)$ and $\tilde{\epsilon}_{d}(N)$ against $N$ for a model with $U / \pi \Delta=6.0$ and $\epsilon_{d} / \pi \Delta=$ -4.0. For the impurity site $N=0$ we take $\tilde{\Delta}(0)=\Delta$, and 
$\tilde{\epsilon}_{d}(0)$ to be the average Hartree-Fock value $\epsilon_{d}+U n_{d} / 2$, where $n_{d}$ is the total occupation value at the impurity site, calculated within the Hartree-Fock theory. This HartreeFock value for $\tilde{\epsilon}_{d}(0)$ is considerably shifted from the bare value $\epsilon_{d}$, but clearly fits the trend in values for $\tilde{\epsilon}_{d}(N)$ for small $N$. If the one-particle excitations can be described by an effective non-interacting Anderson model then $\tilde{\Delta}(N)$ and $\tilde{\epsilon}_{d}(N)$ should be independent of $N$. This can indeed seen to be the case for $N>40$ so for this range these excitations can be described by a model with renormalized parameters $\tilde{\Delta}$ and $\tilde{\epsilon}_{d}$. As the bare parameters correspond to a model in the Kondo regime one sees that in this case $\tilde{\epsilon}_{d} \approx 0$, whereas $\tilde{\Delta} / \Delta$ is small but finite, $\tilde{\Delta}$ being of the order of the Kondo temperature $T_{\mathrm{K}}$.

Once the renormalized parameters $\tilde{\epsilon}_{d}$ and $\tilde{V}$ have been determined the free quasiparticle Hamiltonian can be diagonalized and written in the form

$$
\Lambda^{-(N-1) / 2} \sum_{k=1}^{(N+2) / 2}\left(E_{p, k}(N) p_{k, \sigma}^{\dagger} p_{k, \sigma}+E_{h, k}(N) h_{k, \sigma}^{\dagger} h_{k, \sigma}\right),
$$

where $p_{k, \sigma}^{\dagger}, p_{k, \sigma}$, and $h_{k, \sigma}^{\dagger}, h_{k, \sigma}$, are the creation and annihilation operators for the quasiparticle and quasihole excitations, and $\Lambda^{-(N-1) / 2} E_{p, k}(N)$ and $\Lambda^{-(N-1) / 2} E_{h, k}(N)$ are the corresponding excitation energies relative to the ground or vacuum state $|0\rangle$; the scale factor $\Lambda^{-(N-1) / 2}$ is due to the fact that the energies are calculated for the rescaled Hamiltonian, which is such that $E_{p, k}(N)$ and $E_{h, k}(N)$ for $k=1$ are of order 1 . For the lowest-lying level particle and hole levels, we have $E_{p}(N)=E_{p, 1}(N)$ and $E_{h}(N)=E_{h, 1}(N)$.

From the parameters, $\tilde{\epsilon}_{d}$ and $\tilde{\Delta}$, which determine the free quasiparticle excitations we can immmediately deduce the occupation of the impurity level $n_{\text {imp }}$ at $T=0$ from equation (9), which depends only on the ratio $\tilde{\epsilon}_{d} / \tilde{\Delta}$, the quasiparticle density of states from (7), and the impurity specific heat coefficient $\gamma_{\text {imp }}$ from (8).

To calculate the spin and charge susceptibilities and the low temperature dependence of the conductivity we need to include a quasiparticle interaction term, which for the rescaled model takes the form,

$$
H^{U}(N)=\tilde{U} \Lambda^{(N-1) / 2}: d_{\uparrow}^{\dagger} d_{\uparrow} d_{\downarrow}^{\dagger} d_{\downarrow}:
$$

Asymptotically as $N \rightarrow \infty$ the effect of this term on the low-lying many-particle excitations tends to zero so it is sufficient to calculate the effect of this term to first order in $\tilde{U}$. To this end we need the operator $d_{\sigma}$ expressed in terms of the eigenstates of (15),

$$
d_{\sigma}=\sum_{k=1}^{(N+2) / 2}\left(\psi_{p, k}(-1) p_{k \sigma}+\psi_{h, k}(-1) h_{k \sigma}^{\dagger}\right)
$$

If the lowest two-particle excitation from the ground state for the interacting system for a given $N$ has an energy $E_{p p}(N)$, then we can calculate $\tilde{U}$ by equating the energy difference $E_{p p}(N)-2 E_{p}(N)$ to that calculated using (16) asymptotically in the limit $N \rightarrow \infty$. For finite $N$ we can

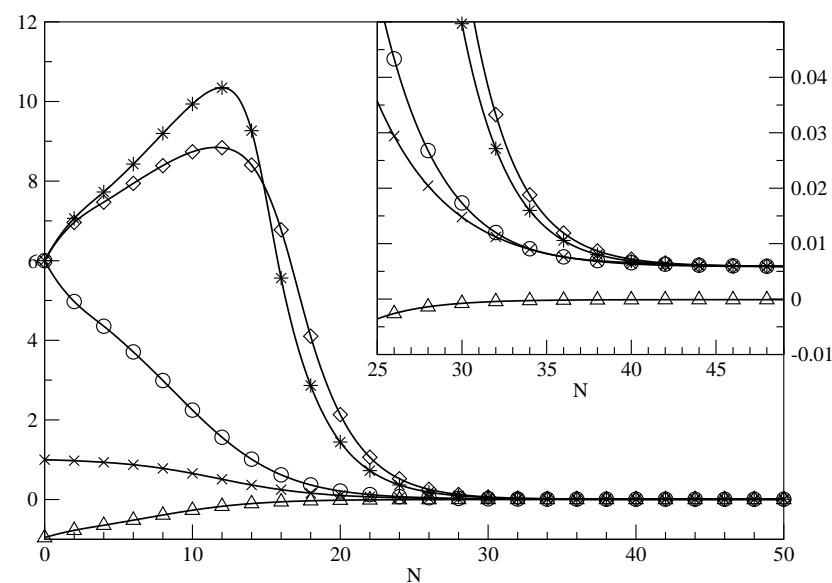

Fig. 1. Plots of the parameters, $\tilde{\Delta}(N) / \pi \Delta$ (crosses), $\tilde{\epsilon}_{d}(N) / \pi \Delta$ (triangles), $\left.\tilde{U}_{p p}(N)\right) / \pi \Delta$ (diamonds), $\tilde{U}_{h h}(N) / \pi \Delta$ (stars) and $\tilde{U}_{p h}(N) / \pi \Delta$ (circles), for the model with bare parameters, $U / \pi \Delta=6.0$ and $\epsilon_{d} / \pi \Delta=-4.0$.

use this equation to define an $N$-dependent renormalized interaction $\tilde{U}_{p p}(N)$,

$E_{p p}(N)-2 E_{p}(N)=\tilde{U}(N) \Lambda^{(N-1) / 2}\left|\psi_{p, 1}^{*}(-1)\right|^{2}\left|\psi_{p, 1}^{*}(-1)\right|^{2}$,

where $\left|\psi_{p, 1}(-1)\right|^{2}$ is given by

$$
\left|\psi_{p, 1}\right|^{2}=\frac{1}{1-\tilde{V}^{2}(N) \Lambda^{(N-1)} g_{00}^{\prime}\left(E_{p}(N)\right)}
$$

where $g^{\prime}{ }_{00}(\omega)$ is the derivative of $g_{00}(\omega)$.

Alternatively we could consider the same procedure for a two hole excitation $E_{h h}(N)$ and in a similar way define an $N$-dependent renormalized interaction $\tilde{U}_{h h}(N)$, or a particle-hole excitation $E_{p h}(N)$ to define a renormalized interaction $\tilde{U}_{p h}(N)$. In this latter case, as a positive $U$ leads to particle-hole attraction, we use $E_{p}(N)+E_{h}(N)-$ $E_{p h}(N)$ on the left-hand side of equation (18).

If these two particle excitations can be described by an effective Anderson model then $\tilde{U}_{p p}(N), \tilde{U}_{h h}(N)$ and $\tilde{U}_{p h}(N)$ should be independent of $N$ and also independent of the particle-hole labels. In figure 1 we also plot $\tilde{U}_{p p}(N)$, $\tilde{U}_{h h}(N)$ and $\tilde{U}_{p h}(N)$ as a function of $N$ for the parameters used earlier, $U / \pi \Delta=6$ and $\epsilon_{d} / \pi \Delta=-4$, with values at $N=0$ corresponding to the unrenormalized interaction $U$. We see that for $N>40$ the values of $\tilde{U}_{p p}(N), \tilde{U}_{h h}(N)$ and $\tilde{U}_{p h}(N)$ do coincide and become independent of $N$ so for the low energy excitations one can define a unique renormalized interaction via $\tilde{U}=\lim _{N \rightarrow \infty} \tilde{U}_{\alpha, \alpha^{\prime}}(N)$, where $\alpha, \alpha^{\prime}=p, h$. What is more, because the model with these chosen parameters corresponds to the Kondo regime, the value of $\tilde{U}$ coincides with the value of $\pi \tilde{\Delta}$, clearly seen in the inset in figure 1, and as can be deduced from equation (6) for $\chi_{c, \text { imp }}=0$.

\subsection{Results for Symmetric Model}

The estimates of $\tilde{\Delta}$ and $\tilde{U}$ for the symmetric model can be checked indirectly from the exact Bethe ansatz results for 


\begin{tabular}{|c|ccc|ccc|}
\hline NRG & \multicolumn{3}{|c|}{$U / \pi \Delta=1$} & \multicolumn{3}{c|}{$U / \pi \Delta=2$} \\
\hline & $\tilde{\Delta} / \Delta$ & $\tilde{U} / \pi \Delta$ & $\mathrm{R}$ & $\tilde{\Delta} / \Delta$ & $\tilde{U} / \pi \Delta$ & $\mathrm{R}$ \\
\hline$\Lambda=2.0$ & 0.6288 & 0.4779 & 1.7600 & 0.2389 & 0.2298 & 1.9620 \\
\hline$\Lambda=2.5$ & 0.6287 & 0.4777 & 1.7599 & 0.2390 & 0.2300 & 1.9621 \\
\hline$\Lambda=3.0$ & 0.6288 & 0.4780 & 1.7601 & 0.2390 & 0.2299 & 1.9619 \\
\hline$\Lambda=3.5$ & 0.6288 & 0.4780 & 1.7601 & 0.2390 & 0.2299 & 1.9620 \\
\hline Exact (BA) & 0.6289 & 0.4780 & 1.7601 & 0.2392 & 0.2301 & 1.9620 \\
\hline
\end{tabular}

Table 1. The renormalized parameters for the symmetric Anderson model for $U / \pi \Delta=1.0$ and $U / \pi \Delta=2.0$ calculated using the NRG and different values of the discretization parameter $\Lambda$. These results are compared with the corresponding values deduced from exact Bethe ansatz results.

this model [12 13]. The exact RPT results for the impurity spin susceptibility and specific heat coefficient at $T=0$ for the symmetric model from (6) and (8) are

$$
\chi_{s, \mathrm{imp}}=\frac{1}{2 \pi \tilde{\Delta}}\left(1+\frac{\tilde{U}}{\pi \tilde{\Delta}}\right), \quad \gamma_{\mathrm{imp}}=\frac{2 \pi}{3 \tilde{\Delta}} .
$$

By equating these to $\chi_{s, \text { imp }}$ and $\gamma_{\text {imp }}$ from the Bethe ansatz we can deduce $\tilde{\Delta}$ and $\tilde{U}$.

Though the discrete model (11) with $\Lambda>1$ and the original model (1) with a continuous spectrum have essentially the same low energy spectrum, i.e. they belong to the same universality class, the dependence of the renormalized parameters on the parameters of the bare model may differ. Such differences can occur in calculations where the high energy excitations, or high cut-offs, are treated differently. This situation occurs for the $N$-fold degenerate models $(U=\infty)$ where the imposition of the high energy cut-off $D^{\prime}$ in the Bethe ansatz calculations for the linear dispersion model differs from the band width $D$ for the conventional model, but a relation between these cut-offs can be found such that the results from the Bethe ansatz calculations can be translated into those for the conventional model [15]. A similar situation applies here. The results can be made equivalent by replacing the bandwidth $D$ of the discrete model by $D A_{\Lambda}$, with $A_{\Lambda}$ is given by

$$
A_{\Lambda}=\frac{1}{2} \frac{1+\Lambda^{-1}}{1-\Lambda^{-1}} \ln \Lambda
$$

where $A_{\Lambda} \rightarrow 1$ in the continuum limit $\Lambda \rightarrow 1$. This result, which is given in the paper of KWW 2 and also used in the paper of Sakai, Shimizu and Kasuya 17, is derived by the requirement that the low energy spectrum of the discrete and continuum model coincide for $U=0$. It means that $V^{2}$ must be increased by a factor $A_{\Lambda}$ when making a comparison with the results of a continuum model with a given $U / \pi \Delta$.

The values of the renormalized parameters $\tilde{\Delta}, \tilde{U}$ and the $\chi / \gamma$ or Wilson ratio, $R=1+\tilde{U} / \pi \tilde{\Delta}[12$, deduced from these are shown in table 1 , where they are compared with those deduced from the Bethe ansatz results. The agreement with the Bethe ansatz results is remarkably good, with errors only of the order of $0.1 \%$, even for values of $\Lambda$ as large as 3.5. It is important for such accurate agreement, particularly for larger values of $\Lambda$ that the $A_{\Lambda}$ factor is taken into account.

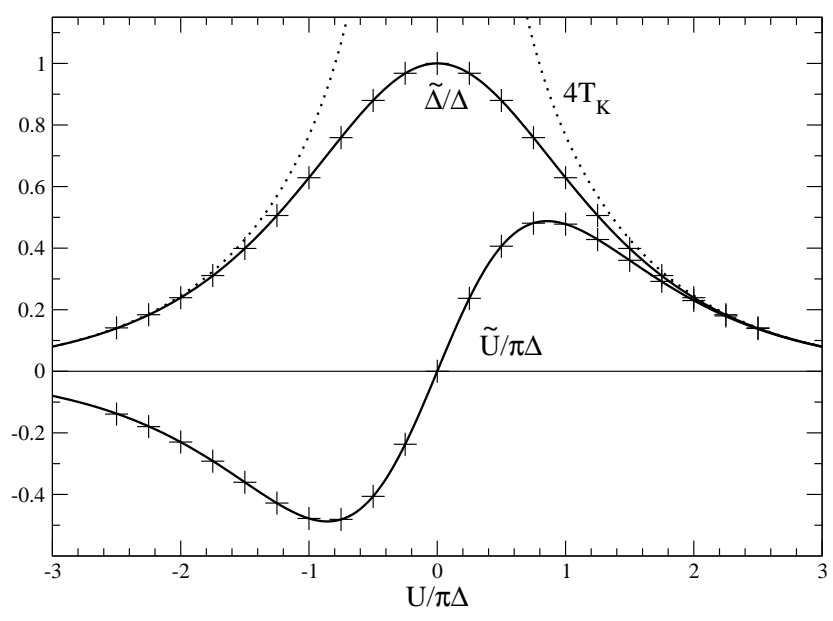

Fig. 2. Renormalized parameters $\tilde{U} / \pi \Delta$ and $\tilde{\Delta} / \Delta$ as a function of $U / \pi \Delta$. The two curves are the results deduced from the Bethe ansatz, and the points marked with a cross are those deduced from NRG calculations with $\Lambda=2.0$. For $|U| / \pi \Delta>2.0$ the two energy scales merge and asymptotically approach $4 T_{\mathrm{K}}$ (dotted line), where $T_{\mathrm{K}}$ is the Kondo temperature.

In Figure 2 we make a more extensive comparison of the results for $\tilde{\Delta}$ and $\tilde{U}$ deduced from the NRG calculations and those deduced from the Bethe ansatz for both positive and negative values of $U$. The agreement is excellent over the whole range. For $U / \pi \Delta>2$ the energy scales merge as the impurity charge becomes localized (Kondo regime) and the impurity charge susceptibility tends to zero. In this limit $\tilde{U} / \pi \tilde{\Delta} \rightarrow 1$ and as a consequence the Wilson ratio $R \rightarrow 2$. The single renormalized energy scale in this regime is the Kondo temperature $T_{\mathrm{K}}$, and $\pi \tilde{\Delta}=\tilde{U}=4 T_{\mathrm{K}}$. In the negative $U$ regime such that $U / \pi \Delta<-2$ a local bipolaron forms such that the spin susceptibility tends to zero, and the charge susceptibility is enhanced by a factor of 2 . In this limit the energy scales again merge such that $\tilde{U} / \pi \tilde{\Delta} \rightarrow-1$. This is because a Kondo effect develops in the isospin channel (doubly occupancy of the impurity site corresponding to up-isospin, and zero occupancy to down-isospin), such that $\pi \tilde{\Delta}=-\tilde{U}=4 T_{\mathrm{K}}$, as the real spin fluctuations are suppressed and $\chi_{s, \text { imp }} \rightarrow 0$. There is a possibility that a Kondo effect of this type might be seen in degenerate atomic gases with the doubly occupied paired states cor- 
responding to molecules [16. The general expression for $T_{\mathrm{K}}$ that covers both Kondo regimes is

$$
T_{\mathrm{K}}=|U|\left(\frac{\Delta}{2|U|}\right)^{1 / 2} e^{-\pi|U| / 8 \Delta+\pi \Delta / 2|U|}
$$

The analysis of the irrelevant terms about the fixed point is not the only way to deduce some of the renormalized parameters from the NRG calculations. If the NRG approach is used also to calculate the dynamics of the impurity model [1819] then $\tilde{\epsilon}_{d}$ and $\tilde{\Delta}$ can be deduced from the self-energy and its derivative for $\omega=0$ and substituted in equation (2). Typical values for $\tilde{\Delta}$ calculated in this way for the symmetric model $\left(\tilde{\epsilon}_{d}=0\right)$ for $U / \pi \Delta=1$ and $U / \pi \Delta=2$ are 0.6155 and 0.2350 , respectively for $\Lambda=2$. They compare well with the Bethe ansatz values given in Table 1. The errors are greater than those deduced from the analysis of the fixed point, of the order of $2-3 \%$, but still quite small, and are of the order to be expected in the calculation of dynamical quantities. Unfortunately the evaluation of $\tilde{U}$ from equation (3) requires a knowledge of the four-vertex $\Gamma_{\uparrow, \downarrow}(0,0,0,0)$ which is very difficult to calculate directly from an NRG calculation, as it involves the Fourier transform of the two-particle Green's function with respect to three independent frequency parameters. Both $\tilde{\Delta}$ and $\tilde{U}$ have, however, been calculated from equations (2) and (3) from perturbation theory to third order in $U$ for the symmetric model 4, and the results are

$$
\tilde{\Delta}=\Delta\left\{1-\left(3-\frac{\pi^{2}}{4}\right)\left(\frac{U}{\pi \Delta}\right)^{2}+\ldots\right\},
$$

and

$$
\tilde{U}=U\left\{1-\left(\frac{\pi^{2}-9}{4}\right)\left(\frac{U}{\pi \Delta}\right)^{2}+\ldots\right\} .
$$

These perturbational results are in good agreement with the exact results in the range $U / \pi \Delta<0.5$; at $U / \pi \Delta=0.5$ the error in $\tilde{\Delta}$ is less than $1.5 \%$ and that for $\tilde{U}$ less than $4 \%$.

We were able to define running renormalized parameters, such as $\tilde{\Delta}(N)$ and $\tilde{\epsilon}_{d}(N)$ as a function of $N$, which raises the possibility that they can be translated into effective parameters appropriate for calculations on an energy scale $\omega_{N}=\eta D \Lambda^{-(N-1) / 2}$ or temperature scale, $T_{N}=\omega_{N}$, where $\eta$ is an appropriately chosen constant of order unity. We do not however have a unique value for $\tilde{U}(N)$. Nevertheless, for the particle-hole symmetric case if we take the value of $\tilde{U}_{p p}(N)\left(=\tilde{U}_{h h}(N)\right)$ as $\tilde{U}(N)$, and translate this, together with $\tilde{\Delta}(N)$ and $\tilde{\epsilon}_{d}(N)$, into parameters appropriate for a temperature scale $T_{N}$, we can generalize the RPT expression for the impurity susceptibility in equation (6) to finite temperatures,

$$
\chi_{\mathrm{imp}}(T)=\tilde{\chi}_{\mathrm{imp}}^{(0)}(T)\left(1+2 \tilde{U} \tilde{\chi}_{\mathrm{imp}}^{(0)}(T)\right)
$$

where $\tilde{\chi}_{\mathrm{imp}}^{(0)}(T)$ is the free quasiparticle contribution to the impurity susceptibility given by

$$
\tilde{\chi}_{\mathrm{imp}}^{(0)}(T)=-\frac{1}{2} \int_{-\infty}^{\infty} \tilde{\rho}_{\mathrm{imp}}(\omega) \frac{\partial f(\omega)}{\partial \omega} d \omega
$$

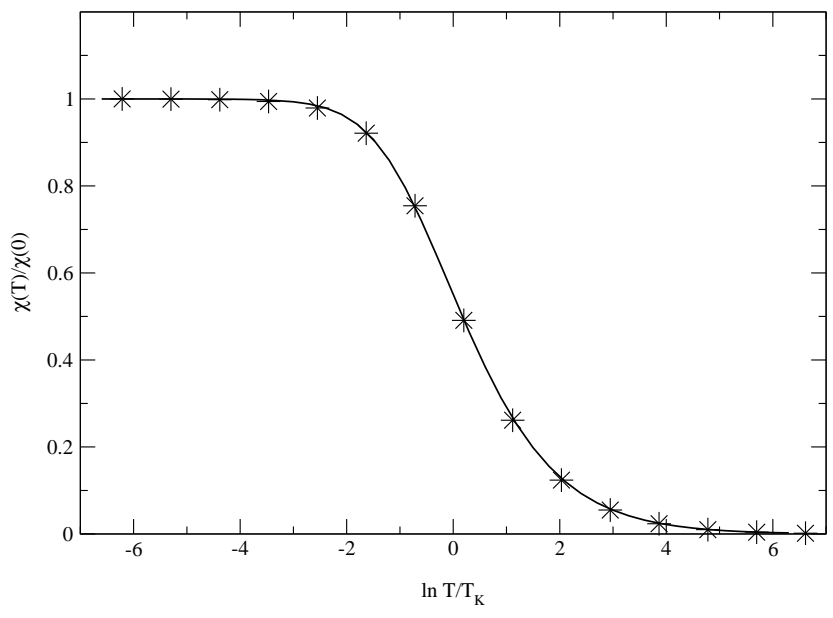

Fig. 3. The temperature dependent impurity susceptibility $\chi_{\text {imp }}(T) / \chi_{\text {imp }}(0)$ (stars) versus $\ln \left(T / T_{\mathrm{K}}\right)$ evaluated from equation (25) for the symmetric model for $U / \pi \Delta=6.0$, compared to the results of the Bethe ansatz solution (continuous curve) for the s-d model given by Tsvelik and Wiegmann [12.

in units of $\left(g \mu_{\mathrm{B}}\right)^{2}$, where $f(\omega)=1 /\left(e^{\omega / T}+1\right)$, and $\tilde{\rho}_{\text {imp }}(\omega)$ is the free quasiparticle density of states given by equation (7). We calculate $\tilde{\chi}_{\text {imp }}^{(0)}(T)$ for the symmetric model in the Kondo regime for $U / \pi \Delta=6.0$ at values of $T_{N}$, using the a renormalized parameters $\tilde{\Delta}\left(T_{N}\right)$ and $\tilde{\epsilon}_{d}\left(T_{N}\right)=0$ in evaluating the free quasiparticle density of states for this energy scale, with $\eta=1.2$ as is used in the NRG evaluation of spectral densities on a scale $\omega_{N}$ (see for example [1819]). We then deduce $\chi_{\mathrm{imp}}(T)$ from equation (25) using $\tilde{U}\left(T_{N}\right)$. In figure 3 we compare the results of this calculation with the Bethe ansatz results for the s-d model given in reference [12. There is quite a remarkable agreement with the exact Bethe ansatz results over this temperature range, and the value of $\chi_{\mathrm{imp}}(T)$ in the extreme high temperature range corresponds to that of the free bare model, $1 / 8 T$. The agreement is much less good if we use $\tilde{U}_{p h}(N)$ for $\tilde{U}(N)$ and, as there is not a unique prediction for this quantity, one cannot place too much reliance on this calculation. However, it does suggest that one might be able to define a renormalized perturbation theory with running coupling constants (this is possible in a magnetic field, with field dependent parameters, as we will demonstrate elsewhere).

\subsection{Results for Asymmetric Model}

We now consider how the renormalized parameters vary as the bare parameters change from one qualitatively different regime of the model to another. We start first of all with the value $\epsilon_{d}=-\pi \Delta=$ constant, and then increase $U$ from $U=0$ to $U=2.7 \pi \Delta$. This takes us from what might be called the full orbital regime to the Kondo regime. The results are plotted in figure 4 . We see that $\tilde{\epsilon}_{d}$ increases at first approximately linearly with $U$ until $U / \pi \Delta \sim 1$, and then slowly increases monotonically through zero at the 


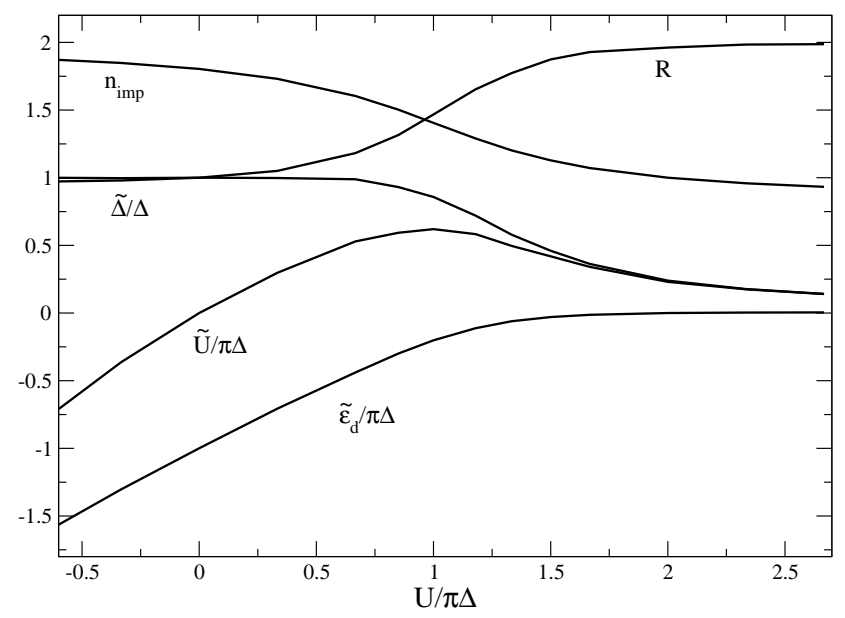

Fig. 4. The renormalized parameters $\tilde{\Delta} / \pi \Delta, \tilde{\epsilon}_{d} / \pi \Delta$, and $\tilde{U} / \pi \Delta$, the Wilson ratio $R=1+\tilde{U} \tilde{\rho}_{d}(0)$, and the impurity occupation value $n_{\text {imp }}$ are plotted for the asymmetric Anderson model for a fixed impurity level $\epsilon_{d}=-\pi \Delta$ as a function $U / \pi \Delta$. Over this range the state of the impurity changes from the full orbital to the Kondo regime.

symmetric point $U / \pi \Delta \sim 2$, remaining very close to zero in the Kondo regime at higher values of $U$. The renormalized resonance width $\tilde{\Delta}$ decreases monotonically over the same range, though only slowly at first in the full orbital regime, and approaches zero in the limit $U \rightarrow \infty$. The quasiparticle interaction $\tilde{U}$ increases at first linearly with $U$, reaching a maximum for $U / \pi \Delta \sim 1$, and then decreases so that its energy scale merges with that for $\tilde{\Delta}$, with $\tilde{U}=\pi \tilde{\Delta}=4 T_{\mathrm{K}}$, as for the symmetric model discussed in the previous section. Over the same parameter range $n_{\text {imp }}$ decreases from an initial value of 1.8 to slightly below unity at $U=2.7 \pi \Delta$. The Wilson ratio or $\chi / \gamma$ ratio, $R=1+\tilde{U} \tilde{\rho}_{d}(0)$, increases from 1 and asymptotically approaches 2 in the Kondo regime. For negative values of $U, \tilde{\epsilon}_{d}$ and $\tilde{U}$ decrease linearly as $U$ decreases and $\tilde{\Delta} / \Delta$ approaches unity. In full orbital regime $U / \pi \Delta<0.5$, including the range with larger negative values of $U$, the Hartree-Fock theory, where $\tilde{\epsilon}_{d}=\epsilon_{d}+U n_{d} / 2$ and $\tilde{\Delta}=\Delta$, constitutes a reasonably good approximation.

It is interesting to compare this behaviour with that for the spectral density $\rho_{d}(\omega)$ of the d-electron Green's function, which can be calculated from the NRG results. Some of the results for $\rho_{d}(\omega)$ over the same parameter range are shown in figure 5(i). Initially there is only a single resonance which moves to higher energies as $U$ is increased. As the Kondo regime is approached this splits into a three peaked structure, the central narrow peak at the Fermi-level being the many-body Kondo resonance. The renormalized Anderson model given by equation (4) describes only a single resonance, but is valid for the low energy behaviour in all the parameter regimes. We see that for smaller values of $U$, and in the negative $U$ regime, $\tilde{\epsilon}_{d}$ tracks at first the lower resonance, increasing monotonically, and in the Kondo regime tracks the Kondo resonance. The precise nature of this tracking is made evident

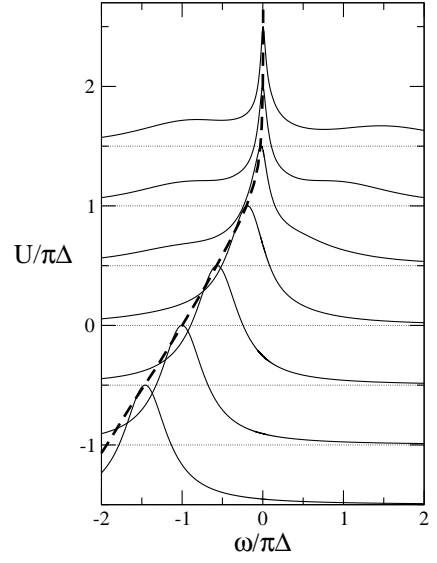

(i)

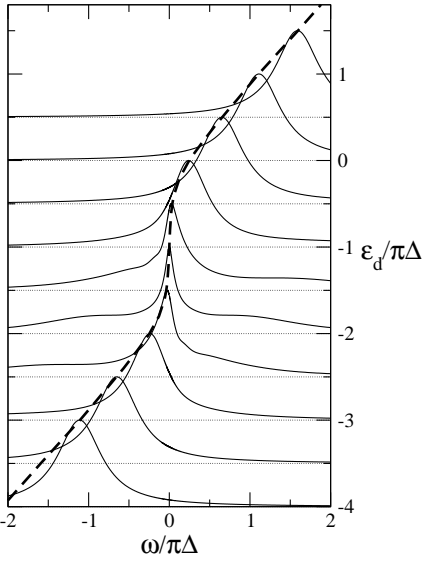

(ii)
Fig. 5. Plots of the spectral density $\rho_{d}(\omega)$ as a function of $\omega$ : (i) for values of $\tilde{U} / \pi \Delta$ with $\epsilon_{d}=-\pi \Delta$ over the same range of values as in figure 4 , and (ii) for values of $\epsilon_{d} / \pi \Delta$ with $U=2 \pi \Delta$ over the same range as in figure 6 . The heights of the maxima in each case are normalized to unity so that the position of the peak can be compared with the values of $\tilde{\epsilon}_{d}$ (dotted line) taken from figure 4 for plot (i), and from figure 6 for plot (ii). The values of $U$ for the curves in (i) can be read off from the left hand scale from the coordinates of the corresponding maxima, and those for $\epsilon_{d}$ in (ii) from the right hand scale.

in figure 5(i) where the curve for $\tilde{\epsilon}_{d}$ taken from figure 4 is plotted with the spectral density results, where the main peak for each $\rho_{d}(\omega)$ has been normalized to unity for the comparison; the maxima of these peaks all lie on the curve. The height of the atomic-like peaks in this plot, which appear at $\omega \sim \epsilon_{d}$ and $\omega \sim \epsilon_{d}+U$ for larger $U$ in the Kondo regime, become somewhat flattened in the normalization as the Kondo peak is so high, making them difficult to see clearly in figure 5 .

In figure 6 we give the renormalized parameters for a complementary scan. In this case we start first of all with $\epsilon_{d}=-3 \pi \Delta$ and $U=2 \pi \Delta$, which is in the almost full orbital regime as $\epsilon_{d}+U<0$, and then increase $\epsilon_{d}$ with a fixed value for $U$ to a final value $\epsilon_{d}=1.5 \pi \Delta$. In doing so we move to a mixed valence regime for $\epsilon_{d}+U \sim 0$, then for $\epsilon_{d}=-\pi \Delta$ the Kondo regime for the symmetric model with $U / \pi \Delta=2$, another mixed valence regime for $\epsilon_{d} \sim 0$, and finally an empty orbital regime for $\epsilon_{d}>\pi \Delta$. In the earlier renormalization group analysis of KWW 2], these parameter regimes are interpreted in terms of essentially three different fixed points, (i) the full (empty) orbital (ii) the strong coupling and (iii) the mixed valent. In the analysis here, however, they are simply different parameter regimes of a single renormalized Anderson model. We see from figure 6 the characteristic features of full and empty orbital regimes, $\tilde{\epsilon}_{d}$ increases linearly with $\epsilon_{d}$, the Wilson ratio $R \sim 1, n_{\text {imp }} \sim 2$ or 0 , and $\tilde{\Delta}$ and $\tilde{U}$ are independent energy scales. In contrast in the Kondo regime, $\tilde{\epsilon}_{d} \approx 0$ and is largely independent of $\epsilon_{d_{2}}$ the Wilson ratio $R \sim 2$, $n_{\text {imp }} \sim 1$, and the energy scales $\Delta$ and $\tilde{U}$ have merged such that $\tilde{U}=\pi \tilde{\Delta}$. The mixed valence regimes appear more as 
cross-over regions between these two types of behaviour, where the independence of the two energy scales $\tilde{\Delta}$ and $\tilde{U}$ emerges. The curve for $\tilde{\epsilon}_{d}$ from figure 6 is plotted in figure 5(ii) with the corresponding results for the spectral density $\rho_{d}(\omega)$, with the height of the peaks normalized to unity. The renormalized level $\tilde{\epsilon}_{d}$ is found to track the single peak in the full orbital regime as it moves up through the intermediate valence regime $\epsilon_{d}+U \sim 0$, where a second lower peak develops near $\omega \sim \epsilon_{d}$, and then it tracks the central narrow resonance at the Fermi-level in the Kondo regime, where a third upper peak develops near $\omega \sim \epsilon_{d}+U$. The reverse process occurs as $\epsilon_{d}$ increases from $\epsilon_{d} \sim-\pi \Delta$, as the side peaks disappear and a single peak emerges in the empty orbital regime. As in figure 5(i) atomic-like side peaks at $\omega \sim \epsilon_{d}$, and $\omega \sim \epsilon_{d}+U$, which appear in the mixed valence and Kondo regimes, are only just discernable due to the normalization of the height of the central resonance. If we concentrate on the mixed valence regime for $\epsilon_{d} \sim 0$ in figure 6 , we see that there is a significant upward shift of $\tilde{\epsilon}_{d}$. This reflects the effective shift in the bare level $\epsilon_{d}$ obtained by Haldane [20] in a poor man's scaling treatment in which the virtual charge fluctuations were eliminated to focus on the mixed valent regime. For the $U=\infty$ limit, the thermodynamic behaviour of the model in this regime was shown by Haldane to depend on the ratio $\bar{\epsilon}_{d} / \Delta$, where $\bar{\epsilon}_{d}=\epsilon_{d}+\Delta / \pi \ln (\pi D / 2 \Delta)$. For the renormalized Anderson model the equivalent ratio is $\tilde{\epsilon}_{d} / \tilde{\Delta}$, so $\bar{\epsilon}_{d}$ is not to be equated with $\tilde{\epsilon}_{d}$ but $\tilde{\epsilon}_{d} / z$. In the mixed valence regime $\epsilon_{d}+U \sim 0$ the Haldane shift can be seen to be in the opposite direction, to lower energies.

In the full and empty orbital regimes again the HartreeFock is a reasonable approximation, particularly when the effective level lies away from the Fermi-level, even though in this case we have a large value of $U$. With $\epsilon_{d}=-3 \pi \Delta$, $U=2 \pi \Delta, n_{d}=1.82$, the Hartree-Fock estimate of the position of the renormalized level $\tilde{\epsilon}_{d} / \pi \Delta=\left(\epsilon_{d}+U n_{d} / 2\right) / \pi \Delta=$ -1.17 is in good agreeement with the renormalization group results, as can be seen in figure 6 . The fact that the quasiparticle interaction $\tilde{U}$ is comparatively large in the full and empty orbital regimes does not imply that the interaction effects on the low energy scale in these regimes are large. On the contrary they are small, as can be seen from the fact that $R$ approaches unity in these two regimes. The reason is that the significant term is the combination $\tilde{U} \tilde{\rho}(0)$, and in these regimes $\tilde{\rho}(0) \rightarrow 0$ as the renormalized level moves well away the Fermi-level, such that $\tilde{U} \tilde{\rho}(0)$ is quite small. A similar situation applies in the case of the $U=\infty N$-fold degenerate Anderson model where slave boson mean field theory is asymptotically exact in the limit $N \rightarrow \infty$. The mean field theory in that case is valid not because $\tilde{U} \rightarrow 0$ as $N \rightarrow \infty$, in fact $\tilde{U}$ remains finite in the limit, but because $\tilde{\rho}(0) \rightarrow 0$ so the combination $\tilde{U} \tilde{\rho}(0) \rightarrow 0$ as $N \rightarrow \infty$ [21].

Though we have derived a consistent picture in the form of a renormalized Anderson model to describe the low energy behaviour within both the NRG and RPT approaches, the two types of calculations involve quite different ways of realising this renormalization; it is interesting to compare and contrast the ways in which they arrive

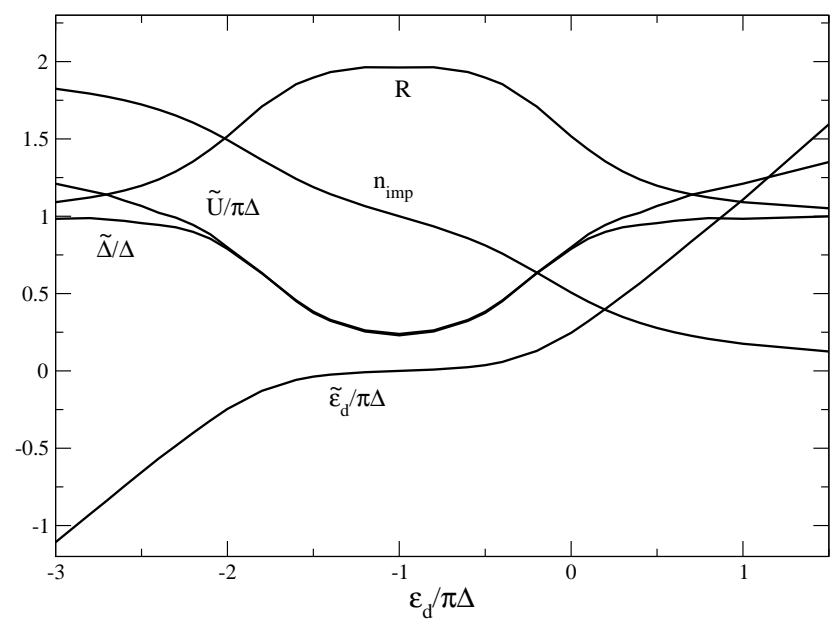

Fig. 6. The renormalized parameters $\tilde{\Delta} / \pi \Delta, \tilde{\epsilon}_{d} / \pi \Delta$, and $\tilde{U} / \pi \Delta$, the Wilson ratio $R$ and the impurity occupation value $n_{\text {imp }}$ are plotted for the asymmetric Anderson model for a fixed value $U=2 \pi \Delta$ as a function of $\epsilon_{d} / \pi \Delta$. On increasing $\epsilon_{d}$ through this range the impurity state passes from the full orbital state, through a mixed valence and Kondo regime, a second mixed valence regime, and to a final empty orbital state.

at the same results. In the NRG approach the excitations at the fixed point are free quasiparticles, and their energy spectrum depends solely on the ratio $\tilde{\epsilon}_{d} / \tilde{\Delta}$, and hence via the Friedel sum rule, equation (9), on the additional charge at the impurity site. The leading irrelevant terms that cause a scattering of these quasiparticles are of the order $\Lambda^{-(N-1) / 2}$ for large $N$ [22] and are of two types; a one-body or hybridization term which determines the width $\tilde{\Delta}$ of the quasiparticle resonance, and a local twobody interaction $\tilde{U}$. This latter term only contributes to states in which two or more quasiparticles are excited.

In the RPT approach the free quasiparticles already contain the effective hybridization, and are characterized by the two parameters, $\tilde{\epsilon}_{d}$, the renormalized impurity level, and $\tilde{\Delta}$, the renormalized width. As in the NRG approach the local two-body interaction term $\tilde{U}$ only comes into play when two or more quasiparticles are excited. This is because the interaction term in the quasiparticle Hamiltonian is normal-ordered with respect to a vacuum corresponding to the interacting ground state, in contrast to the interaction term in the original bare model (11). In the more general renormalization prescription of the RPT,

$$
\tilde{\Sigma}(0,0)=0, \quad \tilde{\Sigma}^{\prime}(0,0)=0, \quad \tilde{\Gamma}_{\uparrow, \downarrow}(0,0,0,0)=\tilde{U},
$$

where $\tilde{\Sigma}(\omega, T)$ is the renormalized self-energy and the renormalized 4 -vertex $\tilde{\Gamma}_{\uparrow, \downarrow}\left(\omega_{1}, \omega_{2}, \omega_{3}, \omega_{4}\right)$ 34, this normal ordering is effectively carried out by the counter terms that ensure these conditions are satisfied.

The two approaches also differ in detail for analytic calculations for the behaviour of the model at higher temperatures and higher energy scales. In the NRG approach the next to leading order irrelevant terms about the fixed point have to be added to the effective Hamiltonian, and some of these will correspond to multiple quasiparticle 
interaction terms. In contrast in the RPT approach, no higher order quasiparticle interaction terms have to be added; though the counter terms have to be fully taken into account order by order in the expansion in $\tilde{U}$. The counterpart of the multiple quasiparticle scattering terms are the higher order Feynman diagrams in the perturbation expansion, which have to be taken into account to probe the higher energy scales. Calculations on these higher energy scales should describe the 'undressing' of the fully renormalized quasiparticles. Calculations to third order in $\tilde{U}$ have been carried out for the particle-hole symmetric model 4 .

\section{Model with a coupling to Phonons}

One possible modification of the impurity model is the inclusion of an interaction with phonons. Such a term could be important when dealing with an impurity with a partially filled $4 \mathrm{f}$ shell, as there can be a change of the ionic volume of the order of $10 \%$ when an electron is removed or added to the $4 \mathrm{f}$ shell due to the adjustment of electrons in the outer $\mathrm{d}$ and $\mathrm{s}$ shells. The simplest type of interaction to consider is a coupling of the occupation of the $\mathrm{d}$ or $\mathrm{f}$ shells to an Einstein phonon of frequency $\omega_{0}$, which is of the form used to study polaronic effects in a tight-binding model by Holstein 23. If we add such a term to the Hamiltonian for the Anderson model we get the Anderson-Holstein model, which we have studied in earlier work 24]. The additional term has the form,

$$
H_{\mathrm{e}-\mathrm{ph}}=g\left(b^{\dagger}+b\right)\left(\sum_{\sigma} d_{\sigma}^{\dagger} d_{\sigma}-1\right)+\omega_{0} b^{\dagger} b
$$

where $b$ and $b^{\dagger}$ are creation and annihilation operators for the phonon modes and a linear coupling has been assumed with a coupling constant $g$. Some insight into the behaviour of this model can be obtained by performing a displaced oscillator transformation, so that the transformed model takes the form,

$$
\begin{aligned}
H^{\prime}= & \hat{U}^{-1} H \hat{U}=\sum_{\sigma} \epsilon_{d, \mathrm{eff}} d_{\sigma}^{\dagger} d_{\sigma}+U_{\mathrm{eff}} n_{d \uparrow} n_{d \downarrow}+ \\
& +\sum_{k, \sigma} V_{\mathbf{k}}\left(e^{\frac{g}{\omega_{0}}\left(b^{\dagger}-b\right)} d_{\sigma}^{\dagger} c_{k \sigma}+e^{-\frac{g}{\omega_{0}}\left(b^{\dagger}-b\right)} c_{k \sigma}^{\dagger} d_{\sigma}\right)+ \\
& +\sum_{k \sigma} \epsilon_{k} c_{k \sigma}^{\dagger} c_{k \sigma}+\omega_{0} b^{\dagger} b,
\end{aligned}
$$

where

$$
\hat{U}=e^{-\frac{g}{\omega_{0}}\left(b^{\dagger}-b\right)\left(\sum_{\sigma} n_{d, \sigma}-1\right)},
$$

and

$$
\epsilon_{d, \mathrm{eff}}=\epsilon_{d}+\frac{g^{2}}{\omega_{0}}, \quad U_{\mathrm{eff}}=U-\frac{2 g^{2}}{\omega_{0}} .
$$

In the large frequency limit $\omega_{0} \rightarrow \infty$, such that $g^{2} / \omega_{0}$ remains finite, the model is equivalent to the original Anderson model with an effective level $\epsilon_{d \text {,eff }}$ and an effective

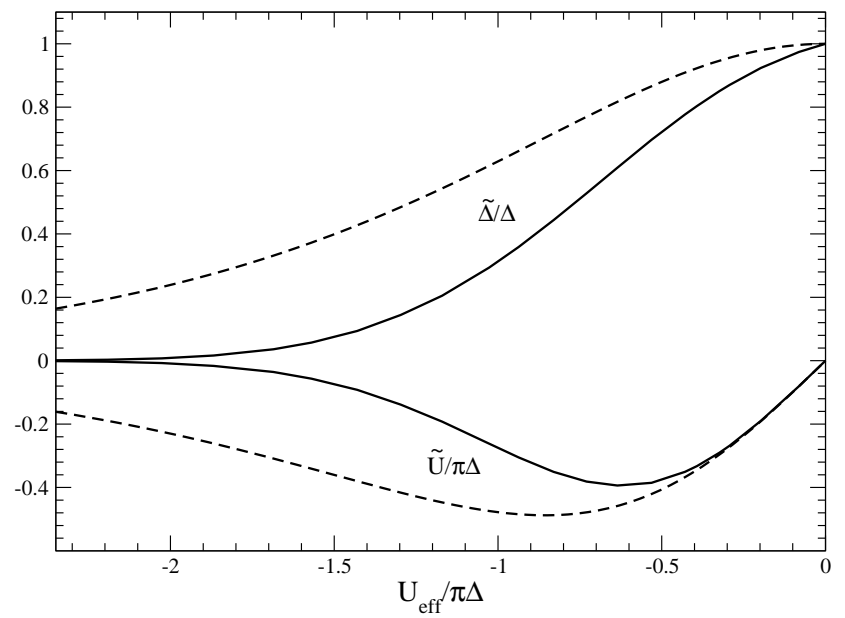

Fig. 7. The values of the renormalized parameters $\tilde{\Delta} / \Delta$ and $\tilde{U} / \Delta$ for the symmetric model with phonons $(U=0)$ plotted as a function of $U_{\text {eff }} / \pi \Delta=-2 g^{2} / \omega_{0} \pi \Delta$. The dotted curves correspond to the limit $\omega_{0} \rightarrow \infty$ ( $U_{\text {eff }}$ finite) and are the same as those for the negative- $U$ model shown in figure 2 . The full lines are for the corresponding curves with $\omega_{0}=0.05$.

local interaction $U_{\text {eff }}$, because in this limit the exponential terms that modify the hybridization term are equal to 1 . When the original Coulomb interaction term $U=0$, the effective interaction is attractive $\left(U_{\text {eff }}<0\right)$, so that in the localized strong coupling regime local bipolarons form, which is equivalent to the formation of a local moment in the spin model, and there is a Kondo effect in the charge (or equivalently isospin) channel.

For the large $\omega_{0}$ limit, such that we can neglect the effect of the exponential terms in the hybridization in the Hamiltonian (29), we require $\omega_{0}$ to be much greater than the conduction bandwidth $D$ [25], which is unrealistic. For the regime $\omega_{0} \ll D$, the physically appropriate one, the exponential terms in the phonon operators in (29) modify the form of the original Anderson model. Nevertheless the low energy fixed point, and the leading irrelevant interaction terms, should correspond to a renormalized Anderson model. The explicit inclusion of the phonon terms should only be necessary in considering higher order irrelevant terms, which contribute only at higher energy scales and higher temperatures. The derivation of the renormalized parameters in equation (2) holds provided that the selfenergy $\Sigma(\omega)$ is analytic at $\omega=0$. We can test this conjecture explicitly by again fitting the lower energy levels obtained in the NRG calculations to a renormalized Anderson model with parameters $\tilde{\epsilon}_{d}, \tilde{\Delta}$ and $\tilde{U}$. We first of all look at the results for the particle-hole symmetric model with $U=0$, where the phonons induce a negative- $U$ term, and assume particle-hole symmetry to compare our results with those obtained in the previous section. In the large $\omega_{0}$ limit these will be identical to those obtained earlier with $U$ being replaced by $U_{\text {eff }}=U-2 g^{2} / \omega_{0}$. For comparison we consider the results for a value of $\omega_{0} \ll D$ and a commensurately smaller value of the coupling $g$, so that it covers a similar range of values of $U_{\text {eff }}$. The results of such a fit- 


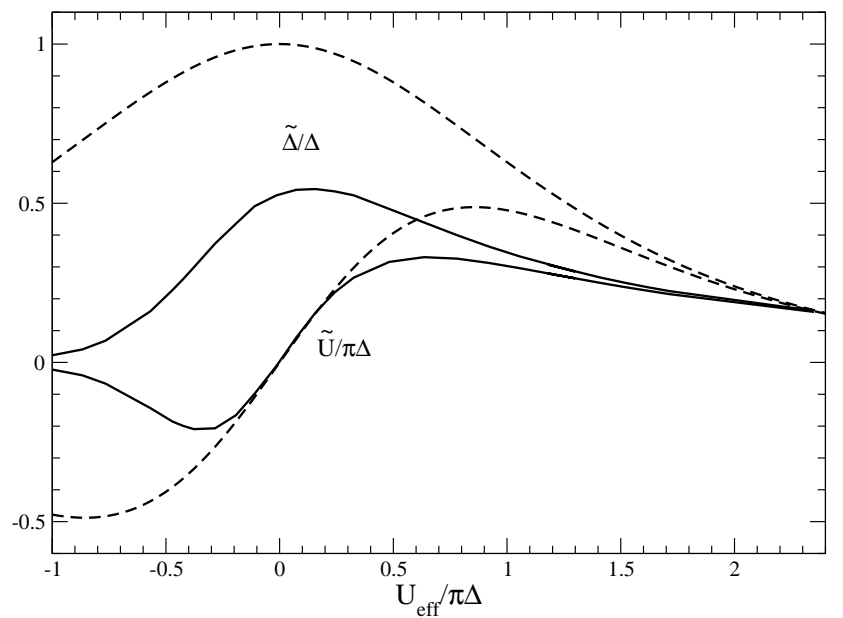

Fig. 8. The values of the renormalized parameters $\tilde{\Delta} / \Delta$ and $\tilde{U} / \Delta$ for the symmetric model with phonons, and $U / \pi \Delta=$ 2.35 , plotted as a function of $U_{\text {eff }} / \pi \Delta=\left(U-2 g^{2} / \omega_{0}\right) / \pi \Delta$. The dotted curves correspond to the limit $\omega_{0} \rightarrow \infty$ ( $U_{\text {eff }}$ finite) and are the same as those for the negative- $U$ model shown in figure 2. The full lines are for the corresponding curves with $\omega_{0}=0.05$.

ting, using the procedure outlined in the previous section, are shown in figure 7 for $\omega_{0} / D=0.05$, and for comparison also the case corresponding to $\omega_{0} \rightarrow \infty$. These values cover the negative $U_{\text {eff }}$ regime as we have $U=0$. We see that, as the coupling $g$ increases, the values of $\tilde{\Delta}$ decrease much more rapidly in the smaller phonon frequency case than in the large $\omega_{0}$ case due to the exponential terms that modify the hybridization in equation (29). The values of $\tilde{U}$ are also commensurately smaller because in the localized or local bipolaron limit, we must have $\tilde{U}=-\pi \tilde{\Delta}$, which is the case for both sets of results in the strong coupling limit.

In the results shown in figure 8 we start with a value of $U / \pi \Delta=2.35$ for which the impurity charge is almost localized and we are in the Kondo regime: as we increase $g$ one effect is to decrease the value of $U_{\text {eff }}$, and in the large $\omega_{0}$ limit this is the sole effect, so that when $U_{\text {eff }}=$ 0 we are left with an unrenormalized width parameter $\tilde{\Delta}=\Delta$. In the low $\omega_{0}$ case the renormalized interaction $\tilde{U}$ changes sign at the same point as the bare parameter $U_{\text {eff }}$ changes sign. This result is somewhat surprising. As the effective interaction induced by the phonons is only valid on the scale $\omega \ll \omega_{0}$, one might have expected that the on-site interaction $U$ would be significantly renormalized on reducing the energy scale to $\omega \sim \omega_{0}$, say to $\bar{U}$, so that $\tilde{U}$ would change sign when $\bar{U}=2 g^{2} / \omega_{0}$, which would be significantly shifted from the condition $U_{\text {eff }}=0$. This is not the case which implies that both the direct and phonon induced interaction terms are renormalized in a similar way.

At the point where $\tilde{U}=0$ there are polaronic effects, which are absent in the large $\omega_{0}$ limit, such that $\tilde{\Delta}$ is significantly renormalized. For the values used here the width is reduced by factor of approximately 2 when $\tilde{U}=0$.

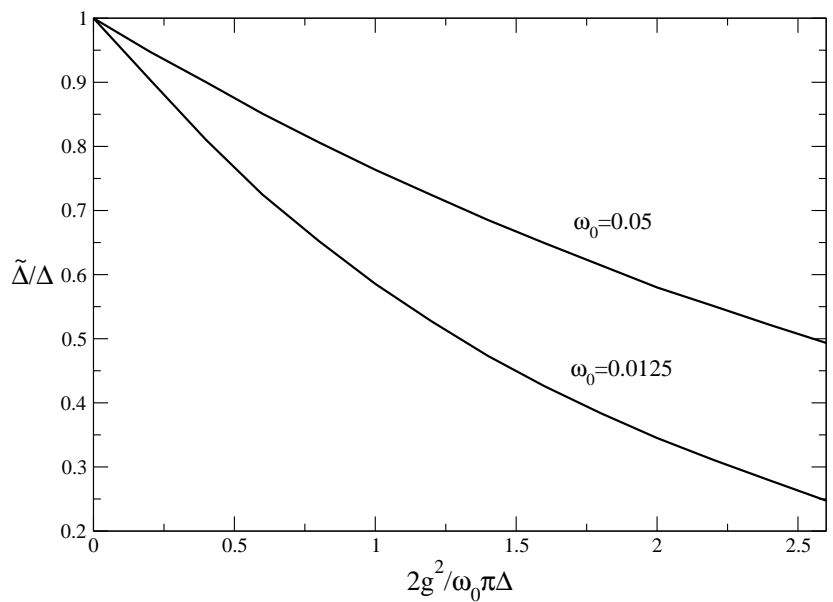

Fig. 9. A plot of the renormalized resonance width $\tilde{\Delta} / \Delta$ for the symmetric model with a phonon coupling $g$ as a function of $2 g^{2} / \omega_{0} \pi \Delta$ with values of $U$ chosen such that $U_{\text {eff }}=U-$ $2 g^{2} / \omega_{0}=0$, for the two cases $\omega_{0}=0.05$ and $\omega_{0}=0.0125$.

In the negative- $U_{\text {eff }}$ regime the behaviour is as in figure 7 , with bipolaronic localization and $\tilde{U}=-\pi \tilde{\Delta}$, with very much reduced values of $\tilde{U}$ and $\tilde{\Delta}$ for the small frequency case as compared with the corresponding values for the large $\omega_{0}$ limit.

In the corresponding spinless model with a coupling to phonons there is no induced attractive on-site interaction, but polaronic effects occur due to the retarded effective potential 23. In the model with spins, we can reveal these polaronic effects if we choose values of $U$ which cancel the attractive interaction that leads to bipolaron formation, $U_{\text {eff }}=U-2 g^{2} / \omega_{0}=0$. We look at the renormalized resonance width for the symmetric model for a range of values of the electron-phonon coupling $g$ with appropriately chosen values of $U$. As a consequence the renormalized quasiparticle interaction $\tilde{U}$ is negligible. The results for the symmetric model are shown in figure 9 for $\omega_{0}=0.05$ and $\omega_{0}=0.0125(D=1)$. It can be seen that the decrease in $\tilde{\Delta}$ with increase of $g$ is greater in the smaller phonon frequency case. In the limiting case $\omega_{0} \rightarrow \infty\left(2 g^{2} / \omega_{0}\right.$ finite) there is no polaronic effect and $\tilde{\Delta} / \Delta=1$ in all cases. Some insight into the behaviour of the asymmetric model $(U=0)$ with a coupling to phonons can be gained by comparing with the negative- $U_{\text {eff }}$ Anderson model as it corresponds to the case $\omega_{0} \rightarrow \infty$. The asymmetric model with negative- $U$ can be mapped into a positive- $U$ symmetric model with a finite magnetic field $H \sim-\epsilon_{d}$ under the interchange of charge and spin; the one-electron spectral density for the isospin up electrons is given by $\rho_{d}(\omega)$ and that for the isospin down by $\rho_{d}(-\omega)$. A finite $\epsilon_{d}$ favours either the doubly occupied state (isospin up) for $\epsilon_{d}<0$, or the empty state for $\epsilon_{d}>0$, so that increasing the value of $\left|U_{\text {eff }}\right|$ has the effect of further increasing the polarization. The results for the renormalized parameters for the asymmetric model with phonons $\left(U=0\right.$ and $\left.\epsilon_{d}=-\pi \Delta\right)$ and finite $\omega_{0}(=0.05)$ which are plotted in figure 10 show a similar trend. As $g^{2}$ increases, or equivalently $U_{\text {eff }}$ decreases, 


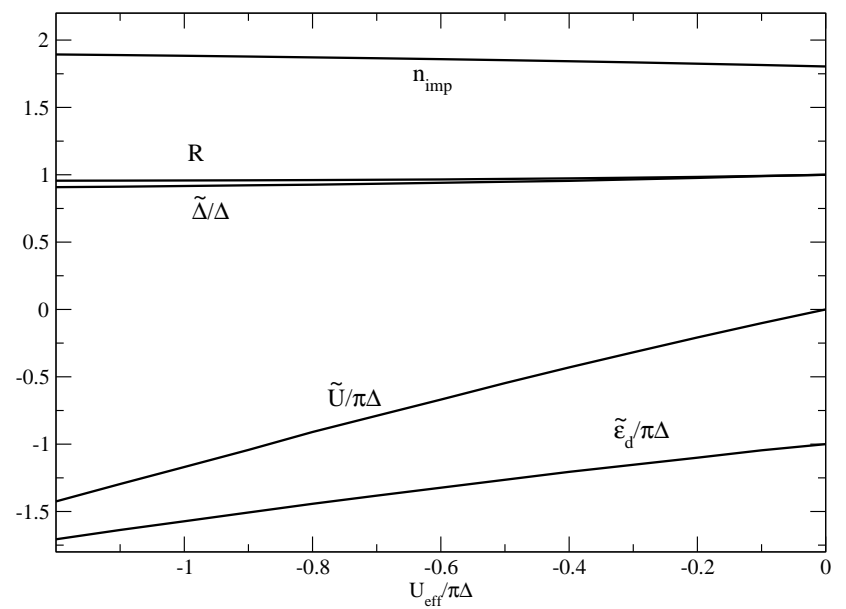

Fig. 10. The renormalized parameters, $\tilde{\Delta} / \pi \Delta, \tilde{\epsilon}_{d} / \pi \Delta$, and $\tilde{U} / \pi \Delta$, the Wilson ratio $R$ and the impurity occupation value $n_{\text {imp }}$ plotted for the asymmetric model $(U=0)$ with a coupling to phonons for a fixed value of $\epsilon_{d}=-\pi \Delta$ as a function of $U_{\text {eff }} / \pi \Delta$ for $\omega_{0}=0.05$.

both $\tilde{\epsilon}_{d}$ and $\tilde{U}$ decrease linearly, and the occupation of the impurity level slowly increases and tends to the value 2 . The interaction effects on the low energy behaviour can be seen to be relatively small because, as $\tilde{\epsilon}_{d}$ moves further from the Fermi-level, the quasiparticle density of states at Fermi-level $\tilde{\rho}_{d}(0)$ decreases and $R \sim 1$. This trend can be clearly seen in the calculated values of the spectral density $\rho_{d}(\omega)$ 24], and $\tilde{\epsilon}_{d}$ tracks the peak in $\rho_{d}(\omega)$. The peaks in $\rho_{d}(\omega)$ become asymmetric for strong electron-phonon coupling, as phonon side-bands excitations are induced on the low energy side of the peak, giving the appearance of a broadened peak. The peak of the renormalized Anderson peak, however, is symmetric with a width $\tilde{\Delta}$ slightly reduced from the bare value $\Delta$, but is quite compatible with the spectra seen in $\rho_{d}(\omega)$ for small $\omega$. The renormalized model has to reproduce the quasiparticle spectrum only in the immediate neighbourhood of the Fermi-level which in this case lies on the higher energy side of the peak in $\rho_{d}(\omega)$.

\section{Model with a Falikov-Kimball Interaction}

Apart from the interaction $U$ between the electrons in the local d- or f-orbitals at the impurity site there will also be a two-body interaction between the electrons in these orbitals and the conduction electrons. Such a term does not appear in the standard Anderson model but a local interaction of this type is included in the Falikov-Kimball model 26. It is the sole two-body interaction term in this model, which was put forward as an appropriate model for investigating valence instabilities in compounds, where the occupation of the localized f-orbitals changes significantly as a result of pressure or alloying (see reference 27] for a recent review). The reason that it is not usually included in impurity models, is not because the interaction is very small, but because it is thought not to play an essential

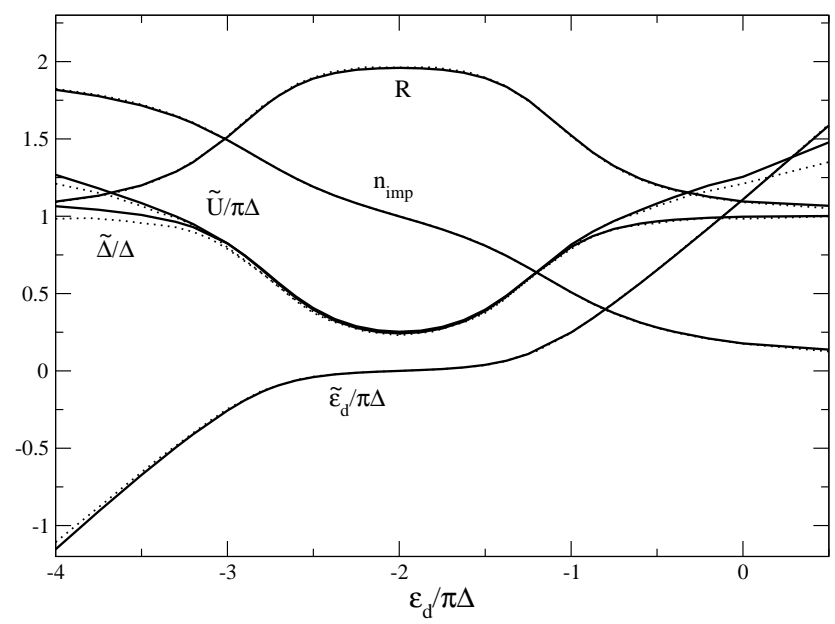

Fig. 11. The renormalized parameters $\tilde{\Delta} / \pi \Delta, \tilde{\epsilon}_{d} / \pi \Delta$, and $\tilde{U} / \pi \Delta$, the Wilson ratio $R$ and the impurity occupation value $n_{\text {imp }}$ are plotted for the Anderson model with an additional Falikov-Kimball interaction $U_{\mathrm{fk}}$. The values of $U$ and $U_{\mathrm{fk}}$ are fixed, such that $U_{\mathrm{fk}}=U / 2=\pi \Delta$, and $\epsilon_{d} / \pi \Delta$ is varied over the range indicated. Also shown as dotted curves are the renormalized values for the Anderson model with $U_{\mathrm{fk}}=0$, corresponding to those shown in figure 6 , but with a shift of $\epsilon_{d}$ such that $\epsilon_{d} \rightarrow \epsilon_{d}-U_{\mathrm{fk}}$. There is a remarkable agreement with these displaced results over this range.

role in understanding the impurity behaviour. This means that its effects can, for the most part, be absorbed as a renormalization of terms that are already within the standard models. We can put this hypothesis to the test by including such a term in the Hamiltonian and then consider what effect it has on the renormalized parameters of the low energy fixed point. In the discrete linear chain version of the Anderson model, equation (11), used in the NRG calculations such a term can be included as an interaction between the impurity and the electron occupation at the first site on the conduction electron chain,

$$
H_{\mathrm{FK}}=U_{\mathrm{fk}} \sum_{\sigma, \sigma^{\prime}} n_{d, \sigma} n_{0, \sigma^{\prime}},
$$

where $U_{\mathrm{fk}}$ denotes the matrix element of the interaction. We add this term to the Hamiltonian in equation (11) and then examine the nature of the low energy fixed point. We take $U=2 \pi \Delta$, as earlier, with $U_{\mathrm{fk}}=U / 2=\pi \Delta$, and take a range of values of $\epsilon_{d}$ to run from the full orbital, through the Kondo, to the empty orbital regime. We can then compare the results with those obtained from a similar study in section 3 .

The results for the renormalized parameters in this study are shown in figure 11 . The low temperature behaviour of the model, $\chi_{\mathrm{s}, \mathrm{imp}}, \chi_{\mathrm{c}, \mathrm{imp}}$ and $\gamma_{\mathrm{imp}}$, can be deduced by substituting the renormalized parameters into equations (6) and (8). There is a clear correspondence with results derived earlier for the Anderson model without this additional term, shown in figure 6 . To examine the correspondence in more detail we have replotted the values from figure 6 in the same figure, using dotted lines, and 
have displaced the curves by a constant shift of $\pi \Delta=U_{\mathrm{fk}}$, such that the original bare level $\epsilon_{d}$ from figure 6 is replaced by an effective bare level $\bar{\epsilon}_{d}=\epsilon_{d}+U_{\mathrm{fk}}$. The agreement between the results with the additional Falikov-Kimball term, $U_{\mathrm{fk}}=\pi \Delta$, and those of the model with $U_{\mathrm{fk}}=0$ and an effective bare level $\bar{\epsilon}_{d}$ are quite remarkable. In the strong correlation regime the differences are less than $5 \%$, and overall less than $10 \%$. As the nature of the low energy fixed point has not been changed by the inclusion of the Falikov-Kimball term, it is not surprising that the low energy behaviour can still be described by a renormalized Anderson model. What is somewhat unexpected in this case is that these renormalized parameters correspond to a constant shift of the bare level over the whole parameter range, and that no adjustment of the bare hybridization term is required.

To examine whether this shift varies or not with $U$, we take $\epsilon_{d}=-2 \pi \Delta$ with $U_{\mathrm{fk}}=\pi \Delta$ so that $\epsilon_{d}+U_{\mathrm{fk}}=-\pi \Delta$, and vary $U$ over the same range as in the results for the pure Anderson model shown in figure 4. If the effective bare level $\bar{\epsilon}_{d}$ is independent of $U$ the results should coincide with those shown in figure 4 . The results of these calculations are shown in figure 12. Again we see that the results are close to those of the Anderson model without this term, with the same shift in the bare $\epsilon_{d}$. The difference in values are less than $4 \%$ for $U / \pi \Delta>1$, and less than $12 \%$ overall. In these results, and those shown in figure 11 , the differences in the values of $n_{\mathrm{imp}}$ are even smaller, less than $1 \%$, over the whole parameter range.

We conclude that the low temperature behaviour of the model with a Falikov-Kimball interaction $U_{\mathrm{fk}}$ in this parameter regime is very similar to that of an Anderson model without this term, but with a shift of $\epsilon_{d}$ to $\bar{\epsilon}_{d}$. The shift is approximately independent of $\epsilon_{d}$ and $U$, and linear for $U_{\mathrm{fk}}$ in the range $0<U_{\mathrm{fk}} / \pi \Delta<2.0$. In this range we found the deviations between the results of the effective model with $\bar{\epsilon}_{d}$ and the model with the Falikov-Kimball term increase linearly with the value of $U_{\mathrm{fk}}$.

In the case of a vanishing hybridization the nature of the fixed point Falikov-Kimball term will be different as it will correspond to an X-ray type of problem, with the localized impurity electron corresponding to a corehole, and the self-energy at $\omega=0$ will be singular [28]. This, however, is a special case, and in general we can expect the self-energy to be regular at $\omega=0$ and the renormalized Anderson model to provide the appropriate low energy model (see also the discussion in Sec. V. B of reference[27]).

\section{Conclusions}

We have shown that the two renormalization approaches to the calculation of the low energy excitations of the Anderson model lead to a consistent picture of the low energy behaviour of the model as described by a renormalized version of the same impurity model. It is interesting to make a comparison of this situation with field theoretic calculations for the $\phi^{4}$ model or quantum electrodynamics (QED). In the perturbation calculations for these models

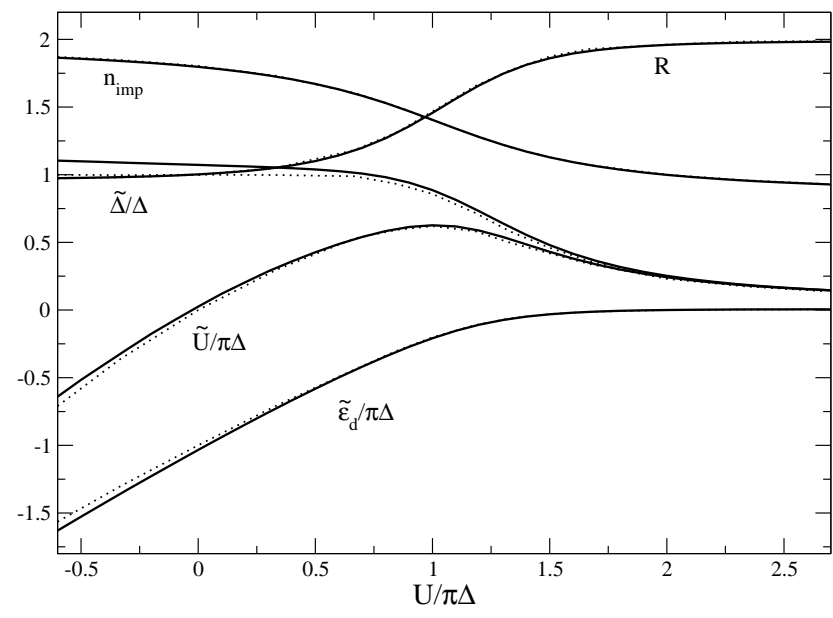

Fig. 12. The renormalized parameters $\tilde{\Delta} / \pi \Delta, \tilde{\epsilon}_{d} / \pi \Delta$, and $\tilde{U} / \pi \Delta$, the Wilson ratio $R$ and the impurity occupation value $n_{\text {imp }}$ for the Anderson model with an additional FalikovKimball interaction $U_{\mathrm{fk}}$, using the same value as for figure 11 . Here, $\epsilon_{d}=-2 \pi \Delta$, and $U / \pi \Delta$ is varied over the range indicated. These results are compared with the corresponding values for the Anderson model with $U_{\mathrm{fk}}=0$ (dotted curves) shown in figure 4 , for which $\epsilon_{d}=-\pi \Delta$. There is again a close agreement with the results of the model without the additional term but with a displaced $\epsilon_{d}$.

infinities arise due to the lack of a high energy cut-off, but the RPT, with the introduction of counter-terms, allows one to by-pass these infinities and perform calculations in terms of a renormalized version of the same model, with particle masses and interactions taken from experiment. The RPT calculations for the Anderson model proceed along similar lines, and the perturbation theory becomes one in terms of the fully dressed quasiparticles and their interactions, in 1-1 correspondence with the original model. Here, however, the renormalizations, though in some situations very large, are not infinite as the conduction band edges provide a high energy cut-off, and the renormalized parameters, $\tilde{\epsilon}_{d}, \tilde{\Delta}$, the analogue of the renormalized masses can be calculated explicitly from equation (2) once the self-energy $\Sigma(\omega)$ is known.

The Anderson model, as a model for impurity systems, neglects many higher energy scale interactions. In including an electron-phonon coupling and a Falikov-Kimball term we have included some of the possible higher energy interaction terms. Explicit calculations have shown that these terms do not change any essential features of the low energy behaviour, which can still be described in terms of a three-parameter renormalized Anderson model, though the renormalized parameters are modified. It is reasonable to assume that if other types of interaction terms are included, such as two-body hybridization terms arising from off-diagonal elements of the Coulomb interaction of the impurity d-electron with the conduction electrons, that the same would hold. The fact that we can describe the various forms of low energy behaviour of this class of non-degenerate impurity models, within the framework of a single renormalized model, gives a unifying perspective. 
It also simplifies the interpretation of experiments on the low temperature behaviour of impurity systems if the results can be analysed in terms of a single three-parameter model.

The non-degenerate Anderson model is not sufficient to describe many impurity systems where the impurity ion has localized incomplete d or f-shells. There are generalizations of the Anderson model that take account of the degeneracy of these configurations, such as the $U=\infty \mathrm{N}$ fold degenerate model for $4 \mathrm{f}$ ionic systems, and the $\mathrm{n}$-fold denenerate models with Hund's rule coupling for d-state ions, and renormalized versions of these models also describe the low energy regime. Explicit calculations of the renormalized parameters have only been carried out in the Kondo regime, with renormalized parameters, such as the renormalized Hund's rule coupling expressed in terms of $T_{\mathrm{K}}$ 1421. Models that have a non-Fermi-liquid low energy fixed point, however, are outside the scope of the present discussion.

Though the extra interactions, such as the coupling to phonons or the Falikov-Kimball term, need not be included explicitly to describe the very low energy behaviour, the question arises as to whether they are needed to describe the behaviour on higher energy scales, or whether can they be simply taken into account via a re-parameterization of the bare model to $\bar{\epsilon}_{d}, \bar{\Delta}$ and $\bar{U}$ [21 29]. There is no simple answer to this question. There are situations where this is formally possible, such as in the model with coupling to phonons in the $\omega_{0} \rightarrow \infty$ limit, where the phonons can be effectively eliminated to give a renormalized bare model, as given in equation (31). However, for $\omega>\omega_{0}$ the real phonon excitations, and not just virtual ones, have to be taken into account explicitly. Even the interaction term $U$ of the bare Anderson model is a renormalized one because its experimental value does not correspond to the Coulomb matrix elements for electrons in localized d-orbitals, but takes into account the many-body relaxation and screening effects of the other electronic shells, when a d or f-electron is removed; so the model is already a renormalized or effective model. This is true for most bare models: which interactions have to be taken into account explicitly is dependent on the energy scales and the type of experiment which is being described. single particle excitations for $\tilde{U}=0$ so that the Green's function at the impurity site $(i=-1)$ is given by

$$
G_{-1-1}(\omega)=\frac{1}{\omega-\epsilon_{d} \Lambda^{(N-1) / 2}-V^{2} \Lambda^{(N-1)} g_{00}(\omega)}
$$

where $g_{00}(\omega)$ is the Green's function at site $i=0$ for the system described by the Hamiltonian $H_{0, N}^{0}$. This Green's function is given in turn by

$$
g_{00}(\omega)=\frac{1}{\omega-\xi_{0}^{2} \Lambda^{(N-1)} g_{11}(\omega)}
$$

and $g_{11}(\omega)$ is the Green's function at site $i=1$ for the system described by the Hamiltonian $H_{1, N}^{0}$. This procedure can be extended to express $g_{00}(E)$ in the form of a continued fraction.

The one-particle excitations $E$ are given by the poles of (33),

$$
E-\epsilon_{d} \Lambda^{(N-1) / 2}-V^{2} \Lambda^{(N-1)} g_{00}(E)=0,
$$

where $g_{00}(E)$ can be expressed in the form,

$$
g_{00}(E)=\sum_{l=1, N+1} \frac{\left|\phi_{l}(0)\right|^{2}}{E-\tilde{E}_{l}(N)},
$$

where $\phi_{l}(0)$ and $\tilde{E}_{l}(N)$ are the eigenvectors and eigenvalues of $H_{0, N}^{0}$.

If $E_{p}^{0}(N)$ and $E_{h}^{0}(N)$ are the lowest particle and hole excitations from the ground state of the Hamiltonian $H_{-1, N}^{0}$ then,

$$
\begin{gathered}
\frac{E_{p}^{0}(N) \Lambda^{-(N-1) / 2}}{V^{2}}-\frac{\epsilon_{d}}{V^{2}}=\Lambda^{(N-1) / 2} g_{00}\left(E_{p}^{0}(N)\right), \\
\frac{-E_{h}^{0}(N) \Lambda^{-(N-1) / 2}}{V^{2}}-\frac{\epsilon_{d}}{V^{2}}=\Lambda^{(N-1) / 2} g_{00}\left(-E_{h}^{0}(N)\right) .
\end{gathered}
$$

If we replace $E_{p(h)}^{0}$ by the corresponding values for the interacting system $U \neq 0, E_{p(h)}$, then they can be used to define $N$-dependent quantities, $\tilde{\epsilon}_{d}(N)$, and $\tilde{\Delta}(N)=$ $\pi \tilde{V}(N)^{2} / 2$

for financial support, and W. Koller for helpful advice. AO wishes to acknowledge support by the Grant-in-Aid for Scientific Research from JSPS. We are also grateful to Y. Shimizu and O. Sakai for helpful discussions, and particularly in relation to the derivation of the correction factor $A_{\Lambda}$ for the discretized model.

\section{A Appendix}

Let the linear chain Hamiltonian (11) of the non-interacting system plus impurity be denoted by $H_{-1, N}^{0}$, and that of the rest of the chain without the impurity starting at site $i$ by $H_{i, N}^{0}$, where $i=0,1,2 \ldots$. We are considering free

$$
\begin{aligned}
& \frac{\pi E_{p}(N) \Lambda^{-(N-1) / 2}}{2 \tilde{\Delta}(N)}-\frac{\pi \tilde{\epsilon}_{d}(N)}{2 \tilde{\Delta}(N)}=\Lambda^{(N-1) / 2} g_{00}\left(E_{p}(N)\right), \\
& \frac{-\pi E_{h}(N) \Lambda^{-(N-1) / 2}}{2 \tilde{\Delta}(N)}-\frac{\pi \tilde{\epsilon}_{d}(N)}{2 \tilde{\Delta}(N)}=\Lambda^{(N-1) / 2} g_{00}\left(-E_{h}(N)\right) .
\end{aligned}
$$

The renormalized parameters $\tilde{\epsilon}_{d}$ and $\tilde{\Delta}$ corresponding to the low energy fixed point are given by $\tilde{\epsilon}_{d}=\lim _{N \rightarrow \infty} \tilde{\epsilon}_{d}(N)$ and $\tilde{\Delta}=\lim _{N \rightarrow \infty} \tilde{\Delta}(N)$, and are determined by the two equations,

$$
\frac{\pi \tilde{\epsilon}_{d}}{2 \tilde{\Delta}}=\lim _{N \rightarrow \infty} \Lambda^{(N-1) / 2} g_{00}\left( \pm E_{p(h)}(N)\right),
$$


and

$$
\frac{\pi}{2 \tilde{\Delta}}=\lim _{N \rightarrow \infty} \frac{\Lambda^{(N-1)}\left(g_{00}\left(E_{p}(N)\right)-g_{00}\left(-E_{h}(N)\right)\right)}{E_{p}(N)+E_{h}(N)} .
$$

The results in equations (41) and (42) are applicable also systems with non-symmetric or non-constant densities of conduction states, with the appropriate form for $g_{00}(\omega)$, which can be evaluated using either equation (36) or (34).

\section{References}

1. K.G. Wilson, Rev. Mod. Phys. 47, 773 (1975).

2. H.R. Krishnamurthy, J.W. Wilkins and K.G. Wilson, Phys. Rev. B 21, 1003 and 1044 (1980).

3. A.C. Hewson, Phys. Rev. Lett. 70, 4007 (1993).

4. A.C. Hewson, J. Phys. Condens.-Matter, 13, 10011 (2001).

5. P.W. Anderson, Phys. Rev. 124, 41 (1961).

6. D.M. Newns and A.C. Hewson, J. Phys. F 10, 2429 (1980).

7. That these results are exact can be deduced from a Ward identity. For details see references 93 .

8. J. Friedel, Can. J. Phys. 54, 1190 (1956); J.M. Langer and V. Ambegaokar, Phys. Rev. 164, 498 (1961); D.C. Langreth, Phys. Rev. 150516 (1966).

9. K. Yamada, Prog. in Theor. Phys., 53, 1970 (1975); 54, 316 (1975).

10. P. Nozières, J. Low Temp. Phys. 17, 31 (1974): Low Temperature Physics Conference Proceedings LT14

11. A. Oguri, Physical Review B 64, 153305 (2001).

12. A.M. Tsvelick and P.B. Wiegmann, Adv. Phys. 32, (1983).

13. B. Horvatić and V. Zlatić, J. Physique 46, 1459 (1985).

14. A.C. Hewson, J. Phys.: Condens. Matter, 5, 6277 (1993).

15. J. Rasul and A.C. Hewson, J. Phys. C 17, 3337 (1984).

16. G.M. Falco, R.A. Duine and H.T.C. Stoof, cond-mat/0304489 (2003).

17. O. Sakai, Y. Shimizu and T. Kasuya Prog. Theor.Phys. Suppl. 108, 73 (1992).

18. O. Sakai, Y. Shimizu and T. Kasuya J. Phys. Soc. Jap. 58, 3666 (1989).

19. T. A. Costi, A.C. Hewson and V. Zlatić, J. Phys.: Condens. Matter 6, 2519 (1994).

20. F.M.D. Haldane, Phys. Rev. Lett., 40, 416 (1978).

21. A.C. Hewson, The Kondo Problem to Heavy Fermions, Chapter 7 (Cambridge Univ. Press, Cambridge, 1993 and 1997).

22. This is a feature of the discrete model, more generally in a continuum case one might expect these terms to fall off as $1 / N$.

23. T. Holstein, Ann. Phys. Lpz., 8, 325 (1959).

24. A.C. Hewson and D. Meyer, J. Phys. Condens.-Matter, 14, 427 (2002).

25. D. Meyer and A.C. Hewson, Acta Physica Polonica B, 34, 769 (2003).

26. L.M. Falikov and J.C. Kimball, Phys. Rev. Letters 22, 997 (1969).

27. J.K. Freericks and V. Zlatić, Rev. Mod. Phys., 75, 1333 (2003).

28. G.D. Mahan, Many Particle Physics (Plenum, New York, 1990).

29. A.C. Hewson, S.C. Bradley, R. Bulla and Y. Ōno, Int. Journ. of Modern Physics B, 15, 2549 (2001). 